\title{
Use of vasoactive/vasodilating drugs for systemic sclerosis (SSC) -related digital ulcers (DUs) in expert tertiary centres: results from the analysis of the observational real-life DeSScipher study
}

\author{
Jelena Blagojevic ${ }^{1}$ - G. Abignano ${ }^{2,3} \cdot$ J. Avouac $^{4} \cdot$ L. Cometi ${ }^{1} \cdot$ M. Frerix ${ }^{5} \cdot$ S. Bellando-Randone ${ }^{1} \cdot$ S. Guiducci ${ }^{1}$.

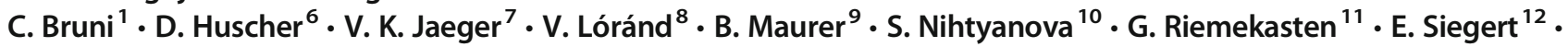

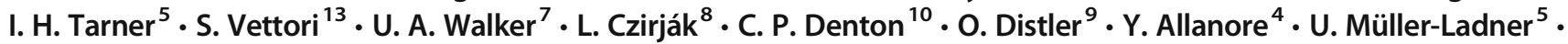 \\ A. Moggi-Pignone ${ }^{14} \cdot$ M. Matucci-Cerinic ${ }^{1} \cdot$ F. Del Galdo ${ }^{3} \cdot$ EUSTAR co-workers $^{2}$
}

Received: 6 January 2019 / Revised: 10 April 2019 / Accepted: 15 April 2019 / Published online: 20 May 2019

(C) International League of Associations for Rheumatology (ILAR) 2019

\begin{abstract}
Introduction DeSScipher is the first European multicentre study on management of systemic sclerosis (SSc), and its observational trial 1 (OT1) evaluated the efficacy of different drugs for digital ulcer (DU) prevention and healing. The aim of this study was to assess current use of vasoactive/vasodilating agents for SSc-related DU in the expert centres by analysing the baseline data of the DeSScipher OT1.

Method Baseline characteristics of patients enrolled in the OT1 and data regarding DU were analysed.

Results The most commonly used drugs, in both patients with and without DU, were calcium channel blockers (CCBs) (71.6\%), followed by intravenous iloprost (20.8\%), endothelin receptor antagonists (ERAs) (20.4\%) and phosphodiesterase 5 (PDE-5) inhibitors $(16.5 \%)$. Of patients, $32.6 \%$ with DU and $12.8 \%$ without DU received two drugs $(p<0.001)$, while $11.5 \%$ with DU and $1.9 \%$ without DU were treated with a combination of three or more agents $(p<0.001)$. Sixty-five percent of the patients with recurrent DU were treated with bosentan and/or sildenafil. However, 64 out of 277 patients with current DU (23.1\%) and 101 (23.6\%) patients with recurrent DU were on CCBs alone.

Conclusions Our study shows that CCBs are still the most commonly used agents for DU management in SSc. The proportion of patients on combination therapy was low, even in patients with recurrent DU: almost one out of four patients with current and recurrent DU was on CCBs alone. Prospective analysis is planned to investigate the efficacy of different drugs/drug combinations on DU healing and prevention.

Key Points

- The analysis of DeSScipher, the first European multicentre study on management of SSc, has shown that the most commonly used vasoactive/vasodilating drugs for DU were CCBs, followed by intravenous Iloprost, ERAs and PDE-5 inhibitors.

- More than half of the patients with recurrent DU received bosentan and/or sildenafil.

- However, the proportion of patients on combination therapy of more than one vasoactive/vasodilating drug was low and almost one out of four patients with current and recurrent $D U$ was on CCBs alone.
\end{abstract}

Keywords Digital ulcer · Management $\cdot$ Systemic sclerosis

\section{Introduction}

Electronic supplementary material The online version of this article (https://doi.org/10.1007/s10067-019-04564-8) contains supplementary material, which is available to authorized users.

Jelena Blagojevic

jelena308@hotmail.com

Extended author information available on the last page of the article
Systemic sclerosis (SSc) is characterised by a complex pathogenesis with tissue fibrosis and vascular remodelling which cause vascular narrowing and loss of capillaries resulting in tissue ischemia [1,2]. Consequently, one of the major complications affecting the extremities are digital ulcers (DUs) that may lead in the most severe cases to gangrene and amputation $[3,4]$. In SSc patients, the presence of DU is a predictor of a 
worse disease course and of a poor survival $[5,6]$. For this reason, the management of DU is a clinical challenge which includes local as well as systemic treatment. In practice, a wide choice of vasoactive and vasodilating drugs, such as calcium channel blockers (CCBs), sildenafil, iloprost and bosentan, are at disposal of the physician [7] but head-tohead comparative studies are not available yet.

DeSScipher is the largest European multicentre observational study with the aim to decipher the optimal management of SSc. It contains five observational trials (OTs) focusing on DU, hand arthritis, interstitial lung disease, pulmonary hypertension and heart disease (https://www.unigiessen.de/ faculties/f11/facilities/desscipher-en?set_language=en). Observational trial no. 1 (OT1) evaluated the effectiveness of different vasoactive/vasodilating drugs for DU prevention and healing.

The aim of this study was to assess the current use of vasoactive/vasodilating therapies employed in expert centres for the treatment of SSc-related DU, by analysing the baseline data of the DeSScipher OT1.

\section{Materials and methods}

OT1 is one of the five trials of the DeSScipher project (ClinicalTrials.gov; OT1 Identifier: NCT01836263). The DeSScipher project was based on use of the EUSTAR (European Scleroderma Trials and Research group) longterm database MEDS (Minimal Essential Data Set) online accessible online at www.eustar-online.org. The structure of the EUSTAR database has been described previously [8].

The names of the EUSTAR co-workers are reported in the Supplementary material 1 .

For the purpose of the DeSScipher observational trials, the MEDS online database was extended and adapted according to the needs of the individual projects. The OT1 specific DeSScipher dataset included more than 30 supplementary clinical items in addition to three items on upper limb lesions contained in the original MEDS online database (digital ulcers, pitting scars on fingertips and gangrene). In particular, the DU section was characterised by the items displayed in Table 1.

The chosen definition of ulcers was the one proposed by the World Sleroderma Foundation (WSF) [9]. DUs were classified according to their main features into DU associated with digital pitting scars (DPS), with calcinosis and with gangrene and DU due to loss of tissue not associated with DPS or calcinosis (Pure DU) [10].

DUs were divided in not recurrent (only one episode) and recurrent DU.

All patients fulfilled American College of Rheumatology (ACR)/European League Against Rheumatism (EULAR) 2013 classification criteria for SSc [11]. Lung involvement was defined when signs of interstitial lung disease were detected at chest X-ray or high-resolution computed tomography (HRCT). Gastro-oesophageal symptoms were defined in MEDSONLINE as follows: esophageal symptoms (dysphagia, reflux) and/or stomach symptoms (early satiety, vomiting). Intestinal symptoms were defined as diarrhoea, bloating and constipation. End-stage organ involvement was defined as at least one of the following features: hyperalimentation required at present, oxygen required at present, left ventricle ejection fraction $>30 \%$ measured at the latest echocardiography and dialysis required at present.

Ethical approval had been obtained from all participating centres' local ethics committees according to Helsinki and its later amendments. Each patient signed a written informed consent form. Moreover, there was an external data monitoring as a part of study quality control.

OT1 data were collected prospectively from March 2013 to November 2016. For the purpose of this study, baseline demographic and clinical characteristics of patients enrolled in OT1 and data regarding DU were analysed. The inclusion criteria of OT1 were current treatment with vasoactive/ vasodilating agents (intravenous iloprost, phosphodiesterase5 (PDE-5) inhibitors such as sildenafil, endothelin receptor antagonists (ERAs) such as bosentan and/or CCBs) and/or ACE inhibitors.

At the time of the analysis (November 2017), clinical data of 1823 patients enrolled into OT1 were stored in the database.

The statistical analysis was performed by SPSS software, version 25 . The results were expressed as mean \pm standard deviation (SD), unless otherwise indicated. For group comparisons of continuous variables, the Mann-Whitney $U$ test was used and, for categorical variables, chi-square test was used. A $p$ value $<0.05$ was considered statistically significant.

\section{Results}

In OT1, clinical data of 1823 enrolled patients were available. Two hundred and seventy-seven (15.2\%) patients presented DU at the enrolment visit, 628 (34.4\%) patients had previous DU while 918 (50.4\%) patients had never experienced DU. Since it has been reported that first DU occurs around 3 years and a half after the onset of SSc [12] and that up to $70 \%$ of patients develop DU in the first 10 years [13], our study population was divided according to disease duration into the following: short disease duration ( $<3$ years) $318(17.5 \%)$, intermediate disease duration ( $\geq 3$ and $<5$ years) 249 (13.7\%), long disease duration (5-10 years) 455 (24.9\%) and very long disease duration (> 10 years) 801 (43.9\%) patients.

Table 2 shows that several demographic and clinical characteristics were significantly different between patients with and without DU and homogeneous disease duration. The features that were significantly different across all groups were 
Table 1 Items of the DeSScipher project items on upper and lower limb DU
OT1 DeSScipher item

\begin{tabular}{ll}
\hline Upper limb DU items & Lower limb ulcer items \\
Pitting scars fingertips & \\
\hline Digital ulcers & Lower limbs: total number of DU \\
DU distal to the PIP & Lower limbs: history of DU \\
DU distal to the PIP: i.v. Iloprost last 3 months or present & Lower limbs: presence infection of DU \\
DU distal to the PIP: recurrent & Lower limbs: gangrene \\
Upper limbs: total number of DU distal to the PIP & Lower limbs: previous amputation \\
Upper limbs: history of DU distal to the PIP & Lower limbs/localisation of DU: patella \\
Upper limbs: presence of infection of DU distal to the PIP & Lower limbs/localisation of DU: malleoli \\
Upper limbs: gangrene & Lower limbs/localisation of DU: calcaneus \\
Upper limbs: previous amputation & Lower limbs/localisation of DU: toes \\
Upper limbs/localisation of DU PIP: fingertip & Lower limbs/localisation of DU: any other \\
& part of leg \\
Upper limbs/localisation of DU PIP: on bony prominence & Lower limbs/localisation of DU: unknown \\
Upper limbs/localisation of DU PIP: unknown & Lower limbs: number of new DU \\
Upper limbs: number of DU defined as loss of tissue & Lower limbs: number of DU healed \\
Upper limbs: number of DU due to calcinosis & Lower limbs: peripheral arterial disease \\
Upper limbs: number of DU due to digital pitting scars & Lower limbs: total number of DU \\
Upper limbs: number DU with unknown origin & Lower limbs: history of DU \\
Upper limbs: number of new DU & Lower limbs: presence infection of DU \\
Upper limbs: number of DU healed & Lower limbs: gangrene \\
Subcutaneous calcinosis of the hands & Lower limbs: previous amputation \\
\hline
\end{tabular}

age, disease subset and modified Rodnan skin score (mRSS) (Table 2).

Among 277 patients with current DU at the enrolment visit, $220(79.4 \%)$ had previous DU, $143(51.5 \%)$ had DU in the last 6 months, while for 57 (20.6\%) patients it was the first DU. Demographic and clinical features and differences between patients with current and previous (healed) DU (items are in italic characters) are shown in Table 3.

Information on recurrent DU was available for $779(86.1 \%)$ patients with DU; 428 (54\%) patients with DU had recurrent DU. Clinical and demographic characteristics of patients with and without recurrent DU are shown in Table 3. Features that were significantly different between two groups are in bold characters.

Pharmacological treatment at the enrolment visit of OT1 is presented in Table 4.

Two hundred-ninety-five $(32.6 \%)$ patients with DU and $116(12.8 \%)$ patients without DU received two vasoactive/ vasodilating drugs $(p<0.001)$, while $104(11.5 \%)$ patients with DU and $18(1.9 \%)$ without DU were treated with a combination of three or four different vasodilating/vasoactive agents $(p<0.000)$. The most commonly used drugs, in both patients with and without DU, were CCBs $(71.6 \%)$, followed by intravenous iloprost (20.8\%), ERAs (20.4\%) and PDE-5 inhibitors $(16.5 \%)$. Bosentan represented $91.4 \%$ of ERAs and sildenafil $92 \%$ of PDE-5 inhibitors prescribed.
Patients (904/1823) (49.6\%) received CCBs alone: 598/ $908(65.6 \%)$ patients without history of DU and 306/905 $(33.8 \%)$ patients with DU (current/previous) $(p<0.001)$. Sixty-four out of 277 patients with current DU (23.1\%) were on CCBs alone compared with 242/628 (38.5\%) patients with previous (healed) DU $(p<0.001)$. Among 428 patients with recurrent DU, 101 (23.6\%) were treated only with CCBs, compared with $159 / 351$ (45.3\%) patients with a single DU episode $(p<0.001)$.

Thirty-six out of 1823 (1.9\%) patients were treated with a combination of prostanoids, PDE-5 inhibitors and ERAs, of which 24/36 (66.7\%) were patients with recurrent DU. Only 13 out of 1823 patients $(0.7 \%)$ were treated with a combination of CCBs, prostanoids, PDE-5 inhibitors and ERAs and 8 of them $(61.5 \%)$ had recurrent DU. Ten percent of patients with DU received bosentan and sildenafil combination treatment, raising to $13 \%$ in patients with recurrent DU.

Drugs that were used significantly more frequently in patients with DU (current or previous) in comparison to those with no history of DU were the following: iloprost (33.8\% vs $8.1 \%, p<0.001)$, ERAs ( $32.7 \%$ vs $8.2 \%, p<0.001)$, bosentan $(31.4 \%$ vs $6.1 \%, p<0.001)$, PDE-5 inhibitors $(23.9 \%$ vs $9.2 \%, p<0.001)$, sildenafil $(22.7 \%$ vs $7.8 \%, p<0.000)$ and combination of bosentan and sildenafil $(9.4 \%$ vs $1.6 \%$, $p<0.001$ ) (for more details see items highlighted in bold green characters in Table 4). 


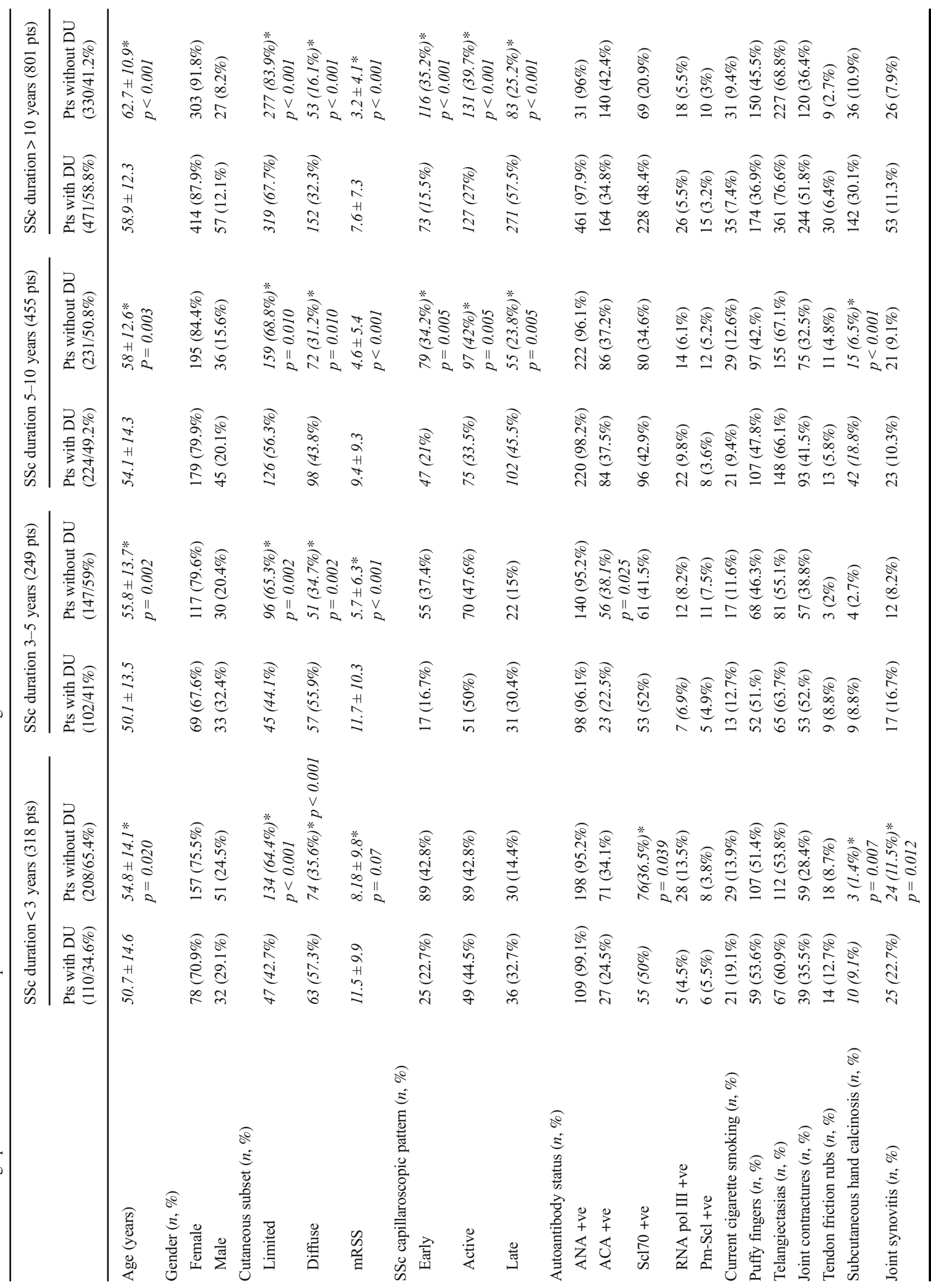


Sixty-five percent of patients with current DU at the enrolment visit were treated with $\mathrm{CCBs}, 50.2 \%$ with intravenous iloprost in and $40.8 \%$ with bosentan. Twenty-seven percent of SSc patients with current DU were on sildenafil and $13 \%$ on sildenafil and bosentan combination treatment. There was a total of 188/277 $(67.5 \%)$ patients treated with bosentan, sildenafil or combination therapy in this group.

Drugs that were used significantly more frequently in patients with current DU compared with patients with previous (healed) DU are highlighted in bold purple characters in Table 4.

Patients with current DU were more frequently on iloprost (50.2\% vs $26.6 \%, p<0.001)$, ERAs $(40.8 \%$ vs $29.1 \%$, $p<0.001$ ), PDE-5 inhibitors (28.2 vs $22 \%, p=0.046)$ and sildenafil ( $20.7 \%$ vs $27.2 \%, p=0.036)$ compared with patients with previous DU. There were significantly more patients on bosentan and on bosentan and sildenafil combination therapy in the group with current DU than in the group with previous (healed) DU ( $40.8 \%$ vs $27.2 \%, p=0.005$ and $13 \%$ vs $7.8 \%$, $p=0.014$, respectively). Of note, the proportion of patients with recurrent DU was higher among patients with current DU compared with those with previous DU ( $79.4 \%$ of vs $44.1 \%, p<0.000$ ).

Patients with recurrent DU were treated most frequently with CCBs $(60.3 \%)$, followed by intravenous iloprost (47.7\%), bosentan (38.1\%), sildenafil (27.2\%) and bosentan and sildenafil combination therapy (13.6\%). There was a total of 279/428 (65.2\%) patients on sildenafil, bosentan or combination therapy in this group.

Drugs that were used significantly more frequently in patients with recurrent DU in comparison to those with a single DU episode are highlighted in bold blue characters in Table 4.

Patients with recurrent DU received iloprost $(47.7 \%$ vs $20.2 \%, p<0.000)$, ERAs (39\% vs $27.4 \%, p<0.001)$ and bosentan $(38.1 \%$ vs $25.4 \%, p<0.000)$, PDE-5 inhibitors (28.7\% vs $18.8 \%, p<0.001)$, sildenafil $(27.2 \%$ vs $18.2 \%$, $p=0.003)$ and combination of sildenafil and bosentan (13.6\% vs $5.4 \%, p<0.001)$, more frequently than patients with single DU episode.

When patients with bosentan and sildenafil combination therapy were excluded from bosentan and sildenafil treatment groups respectively, there were no differences among patient with and without recurrent DU treated with sildenafil or bosentan alone $(24.5 \%$ vs $19.9 \%$ and $13.6 \%$ vs $12.8 \%$, respectively).

Of note, $25 \%$ of patients without recurrent DU were on bosentan treatment, alone or in combination with sildenafil. Only $4.8 \%$ of patients in this group had pulmonary hypertension $(\mathrm{PH})$; therefore, this vasoactive therapy was most likely prescribed for peripheral vasculopathy.

Forty-six percent of patients were on anti-platelet treatment, regardless the history of DU, reaching $53 \%$ in patients with current DU. 
Table 3 Demographic and clinical features of patients with and without current and recurrent DU

\begin{tabular}{|c|c|c|c|c|}
\hline & $\begin{array}{l}\text { Pts with current } \\
\text { DU (277) }\end{array}$ & $\begin{array}{l}\text { Pts with previous (healed) } \\
\text { DU (628) }\end{array}$ & $\begin{array}{l}\text { Pts with recurrent } \\
\text { DU (428) }\end{array}$ & $\begin{array}{l}\text { Pts without recurrent } \\
\text { DU (351) }\end{array}$ \\
\hline Age (years) & $53.4 \pm 13.3$ & $\begin{array}{l}56.8 \pm 13.7 * \\
(p<0.001)\end{array}$ & $55.2 \pm 13.9$ & $59.6 \pm 13.6$ \\
\hline \multicolumn{5}{|l|}{ Gender $(n, \%)$} \\
\hline Female & $218(78.8 \%)$ & $518(82.5 \%)$ & $354(82.7 \%)$ & $289(82.3 \%)$ \\
\hline Male & $59(21.3 \%)$ & $110(17.5 \%)$ & $74(17.3 \%)$ & $62(17.7 \%)$ \\
\hline \multicolumn{5}{|l|}{ Cutaneous subset $(n, \%)$} \\
\hline Limited & $143(51.6 \%)$ & $393(62.6 \%) *(p=0.003)$ & $223(52.1 \%)$ & $289(82.3 \%)^{*}(p<0.001)$ \\
\hline Diffuse & $134(48.4 \%)$ & $235(37.4 \%) *(p=0.003)$ & $170(17.3 \%)$ & $62(17.7 \%)^{*}(p<0.001)$ \\
\hline RP duration (years) & $15.1 \pm 11.4$ & $15.1 \pm 12$ & $15.5 \pm 10.9$ & $14.7 \pm 12.5$ \\
\hline SSc duration (years) & $12.1 \pm 8.9$ & $12.2 \pm 9.2$ & $12.4 \pm 8.4$ & $11.9 \pm 10.1$ \\
\hline mRSS & $11.7 \pm 9.7$ & $7.8 \pm 8^{*}(p<0.001)$ & $10.3 \pm 8.9$ & $6.2 \pm 7.6 * p<0.001$ \\
\hline \multicolumn{5}{|l|}{ SSc capillaroscopic pattern $(n, \%)$} \\
\hline Early & $69(24.9 \%)$ & $236(37.6 \%) *(p=0.002)$ & $26(6.1 \%)$ & $46(13.1 \%) * p<0.001$ \\
\hline Active & $39(14.1 \%)$ & $119(18.9 \%)$ & $53(12.4 \%)$ & $73(20.8 \%) * p<0.001$ \\
\hline Late & $169(61 \%)$ & $273(43.5 \%) *(p=0.002)$ & $122(28.5 \%)$ & $63(17.9 \%) * p<0.001$ \\
\hline \multicolumn{5}{|l|}{ Autoantibody status $(n, \%)$} \\
\hline ANA +ve & $274(98.9 \%)$ & $612(97.5 \%)$ & $396(92.5 \%)$ & $325(92.6 \%)$ \\
\hline $\mathrm{ACA}+\mathrm{ve}$ & $82(29.6 \%)$ & $221(35.1 \%)$ & $118(27.6 \%)$ & $120(38.5 \%) * p=0.034$ \\
\hline Scl70 +ve & $155(55.9 \%)$ & $276(43.9 \%)$ & $201(47 \%)$ & $127(36.2 \%) * p=0.002$ \\
\hline RNA pol III +ve & $15(5.4 \%)$ & $47(7.5 \%)$ & $13(3 \%)$ & $15(4.3 \%)$ \\
\hline $\mathrm{Pm}-\mathrm{Scl}+\mathrm{ve}$ & $12(4.3 \%)$ & $25(4.0 \%)$ & $10(2.3 \%)$ & $8(2.3 \%)$ \\
\hline Current cigarette smoking $(n, \%)$ & $34(12.3 \%)$ & $\begin{array}{l}56(8.9 \%) * \\
p=0.031\end{array}$ & $38(8.9 \%)$ & $37(10.5 \%)$ \\
\hline Puffy fingers $(n, \%)$ & $114(41.2 \%)$ & $226(36 \%)$ & $163(38.1 \%)$ & $140(39.9 \%)$ \\
\hline Telangiectasia $(n, \%)$ & $213(76.8 \%)$ & $420(66.9 \%) *(p=0.013)$ & $310(72.4 \%)$ & $225(64.1 \%) * p=0.003$ \\
\hline Joint contractures $(n, \%)$ & $158(57 \%)$ & $270(43 \%) *(p<0.001)$ & $245(57.2 \%)$ & $126(35.9 \%)^{*} p<0.001$ \\
\hline Tendon friction rubs $(n, \%)$ & $30(10.8 \%)$ & $39(6.2 \%) *(p=0.015)$ & $42(9.8 \%)$ & $18(5.1 \%) * p=0.014$ \\
\hline Subcutaneous hand calcinosis $(n, \%)$ & $77(27.8 \%)$ & $125(20 \%) *(p=0.014)$ & $104(24.3 \%)$ & $60(17.1 \%)^{*} p=0.007$ \\
\hline Joint synovitis $(n, \%)$ & $40(14.4 \%)$ & $80(12.7 \%)$ & $52(12.1 \%)$ & $38(10.8 \%)$ \\
\hline Gastro-esophageal symptoms $(n, \%)$ & $245(88.5 \%)$ & $432(68.8 \%)$ & $300(70.1 \%)$ & $232(66.1 \%)$ \\
\hline Intestinal symptoms $(n, \%)$ & $84(30.3 \%)$ & $203(32.3 \%)$ & $130(30.4 \%)$ & $110(31.5 \%)$ \\
\hline Lung fibrosis $(\mathrm{Rx}$ or HRCT) $(n, \%)$ & $196(70.7 \%)$ & $399(63.6 \%)$ & $268(62.6 \%)$ & $166(47.3 \%)^{*} p<0.001$ \\
\hline Pulmonary hypertension at RHC $(n, \%)$ & $12(4.3 \%)$ & $30(4.8 \%)$ & $19(4.4 \%)$ & $17(4.8 \%)$ \\
\hline Ventricular arrhythmias $(n, \%)$ & $11(3.8 \%)$ & $20(3.2 \%)$ & $5(1.2 \%)$ & $3(0.9 \%)$ \\
\hline Renal crisis $(n, \%)$ & $2(0.7 \%)$ & $11(1.8 \%)$ & $5(1.5 \%)$ & $4(1.1 \%)$ \\
\hline End-stage organ involvement $(n, \%)$ & $6(2.2 \%)$ & $28(4.5 \%)$ & $16(3.7 \%)$ & $13(3.7 \%)$ \\
\hline
\end{tabular}

Pts patients, $R P$ Raynaud's phenomenon, $m R S S$ modified Rodnan skin score, $A N A+v e$ antinuclear antibodies positive, $A C A+v e$ anti-centromere antibodies positive, $S c l 70+v e$ anti-Scl70 (anti-topoisomerase) antibodies positive, $R N A$ pol III + ve anti-RNA polymerase III antibodies positive, $P m$ $S c l+v e$ anti-Pml-Scl antibodies positive, $R x \mathrm{X}$-ray, HRCT high-resolution chest tomography, $R H C$ right heart catheterisation. The values that were significantly different between patients with current and previous DU are highlighted in italic characters. The values that were significantly different between patients with recurrent and not recurrent DU are highlighted in bold characters

There were no significant differences in the use of steroids and/or immunosuppressants in patients with and without DU. At least one out of two patients was treated with immunosuppressants and more than $40 \%$ of patients were on corticosteroids, regardless of the presence of DU. However, patients with the diffuse cutaneous subset received immunosuppressive therapy more frequently than patients with limited cutaneous SSc $(69.4 \%$ vs $41.5 \%, p<0.000)$. The most frequently used immunosuppressants were mycophenolate/mycophenolic acid (279 $(33.1 \%))$ and methotrexate $(273(32.4 \%))$, followed by azathioprine $(162(19.2 \%))$. 


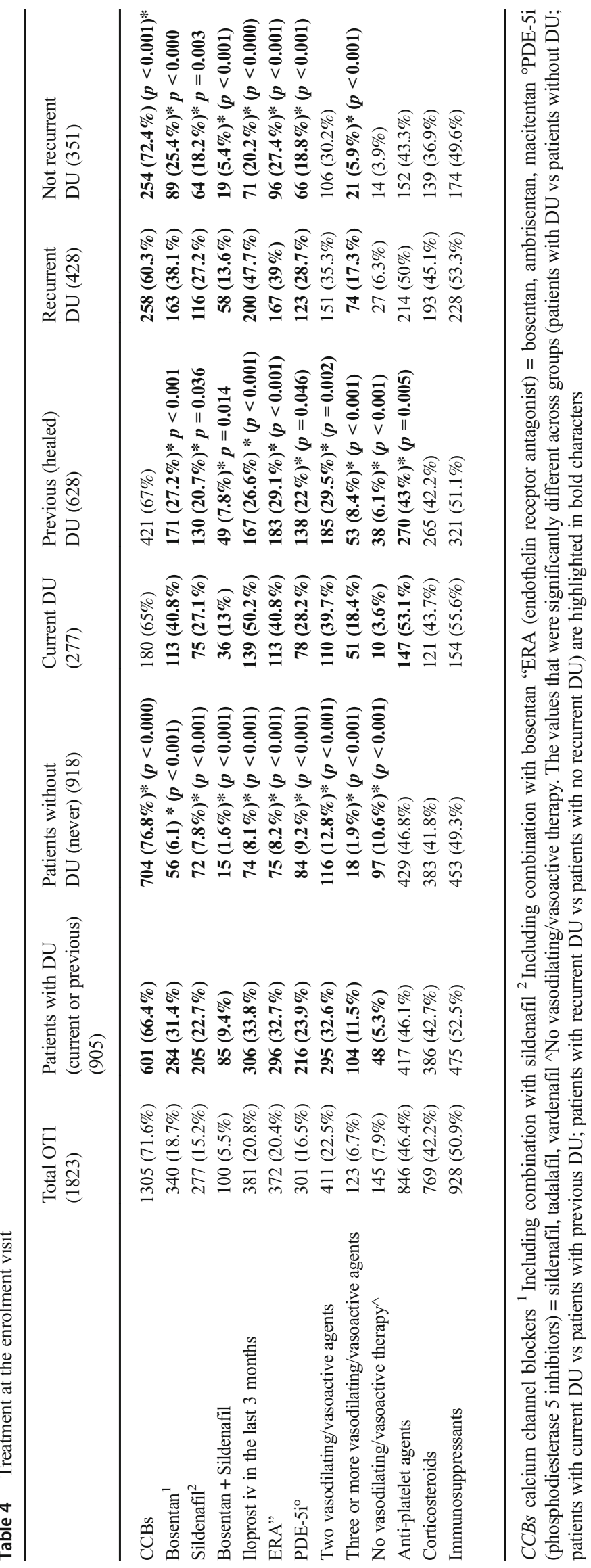




\section{Discussion}

This is the first study that describes the current use of vasoactive/vasodilating agents for SSc-related DU in expert centres, including more than 1800 patients with DU, enrolled in a large multicentre cohort. The observational design of the DeSScipher project with real-life data reflects current clinical practice in tertiary centres for SSc management across Europe.

The prevalence of DU in this study cohort was $49 \%$, higher than recently reported in a large EUSTAR cohort in which $34 \%$ of SSc patients had a history of DU [5]. This is related to the fact that OT1 was designed to be focused on use of vasoactive/vasodilating drugs for DU; therefore, patients with severe peripheral vasculopathy were recruited.

The proportion of patients with DU (current or previous) increased with SSc duration as expected. After stratifying the patients for disease duration, patients with DU (current or previous) were younger, had more frequently a diffuse cutaneous subset and had higher mRSS compared with patients without DU. Diffuse disease subset $[8,14-18]$ and high mRSS $[15-18,19]$ have already been identified as strong risk factors for DU in SSc in large cohort studies.

Interestingly, there were no significant differences in the prevalence of PH in patients with DU compared with those without DU history, differently from what we expected. In addition, smoking habit was associated with current DU, but not DU history, differently from what suggested is by a previous systematic review [16] and a recent EUSTAR-based prospective study [20].

In our study, $94.7 \%$ of patients with DU (current and/or previous) and $89.4 \%$ of patients without DU history were treated with CCBs, iloprost, ERAs and/or PDE-5 inhibitors. The high proportion of treated subjects was correlated to the inclusion criteria. Since the prevalence of PH in these two groups was $4.9 \%$ and $3.3 \%$ respectively, these vasoactive/vasodilating drugs were given mainly for peripheral vasculopathy.

The most commonly used drugs in our cohort, in both patients with and without DU, were CCBs, followed by intravenous iloprost, ERAs and PDE-5 inhibitors. A similar distribution was reported in a large German cohort [21]. On the contrary, in the Canadian cohort only, a very small proportion of patients was on iloprost or bosentan $(<10 \%)$, but at the time when the article was published, these drugs had not been approved for DU in Canada [22].

Patients without a history of DU were treated more frequently with CCBs alone and less frequently with intravenous iloprost and PDE-5 inhibitors, compared with patients with current and/or previous DU. This clearly reveals the intention to treat patients with second line drugs in the presence of DU. In fact, the EULAR recommendations indicate the use of CCBs, usually oral nifedipine, as a first-line treatment for SSc-related Raynaud's phenomenon (RP) [7].

Regarding patients with current DU, half of them were treated with intravenous iloprost, alone or in combination with oral drugs (CCBs, PDE-5 inhibitors, ERAs), while half of them received only oral therapy. In addition, $67 \%$ of the patients with current DU were on bosentan, sildenafil or bosentan and sildenafil combination treatment.

The EULAR recommendations suggest that PDE-5 inhibitors should be considered for the treatment of DU and advise intravenous iloprost in patients with DU not responding to oral therapy [7]. The use of bosentan is recommended in patients with multiple DUs despite treatment with other vasodilators, such as CCBs, PDE-5 inhibitors and iloprost, to prevent the development of new DUs [7].

In our cohort, $28 \%$ of patients with current DU received PDE-5 inhibitors, compared with $40 \%$ and $50 \%$ of patients treated with bosentan and iloprost respectively. This relatively lower usage of PDE-5 inhibitors is probably related to the fact that this drug class has not yet been approved for DU management in Europe.

Patients with recurrent DU were on bosentan and/or sildenafil in $65 \%$ of cases. They were treated more frequently with these two drugs compared with patients with single a DU episode, but surprisingly, when bosentan was considered alone (not in association with sildenafil), no difference was observed between patients with and without recurrent DU.

In addition, $25 \%$ of patients without recurrent DU were on bosentan prescribed for peripheral vasculopathy, alone or in combination with sildenafil, despite the lack of approved drug indication.

Our results indicate that a relatively low proportion of patients were on combination treatment of two or more vasodilating/vasoactive agents: $39 \%$ and $18 \%$ of patients with current DU and $35 \%$ and $17 \%$ of patients with recurrent DU received two and three or more drugs respectively. This may reflect the concern of prescribing physicians about the potential drug-related side effect that may be enhanced using different classes of drugs concomitantly.

On the other hand, $23 \%$ of patients with current and recurrent DU were on CCBs alone, indicating that around one out of four patients with DU are probably still undertreated, even in expert centres.

Of note that half of the patients were on anti-platelet treatment, regardless the history of DU. This probably reflects the perceived importance of platelets' role in the pathogenesis of SSc-related vasculopathy [23], although no study has addressed the use of these drugs for DU or for other SSc manifestations.

In addition, our results show that more than half of the patients were on immunosuppressive treatment and more than $40 \%$ received steroid therapy, regardless the presence of DU.

This study has a number of limitations. The main limitation is represented by the fact that the study included only patients on vasoactive/vasodilating therapies currently in use for peripheral vasculopathy and DU or patients on ACE inhibitors. Other major limitations are the cross-sectional design (the analysis of the OT1 baseline data only) and the fact that the 
participants were represented by the expert tertiary centres that may lead to overestimation of specific drug use in clinical practice. No sub-analysis for different PDE-5i, ERAs and prostanoids, other than sildenafil, bosentan and iloprost, was done, due to the small number of patients treated with these agents. We did not perform sub-analysis for use of ACE inhibitors or for different types of CCBs.

Most importantly, the use of specific combinations of different vasoactive/vasodilating agents, other than sildenafil and bosentan, was not assessed, due to the large number of possible drug associations. In addition, the use of other drugs, such as pentoxifylline and nitrates, was not investigated in this study.

We did not assess the use of different agents for the treatment of different subtypes of DU with possibly diverse pathogenesis (pure DU, DU due to DPS or calcinosis). Finally, we investigated only pharmacological systemic treatment for DU, and not local therapies, which may vary even across the expert centres and impact DU outcome.

\section{Conclusions}

Our study shows that CCBs are still the most commonly used agents for DU management in SSc.

In the expert centres, the proportion of patients on combination therapy with more than one vasodilating/vasoactive drug was still low, even in patients with recurrent DU: almost one out of four patients with current and recurrent DU was on CCBs alone. Prospective analysis is planned to investigate the efficacy of different drugs/drug combinations on DU healing and prevention.

Funding information This study, as part of the DeSScipher project, was supported by the European Community's Framework Programme 7 (FP7-HEALTH-2012.2.4.4-2 Observational trials in rare diseases; grant agreement no. 305495). The authors report personal fees and nonfinancial support (to LV) from the European Union Seventh Framework Program 7 (FP7-HEALTH-2012.2.4.4-2 Observational trials in rare diseases]; grant agreement no. 305495).

\section{Compliance with ethical standards}

Disclosures None.

Ethical approval Ethical approval had been obtained from all participating centres' local ethics committees, according to the declaration of Helsinki and its later amendments. Each patient signed a written informed consent form.

\section{References}

1. Varga J, Trojanowska M, Kuwana M (2017) Pathogenesis of systemic sclerosis: recent insights of molecular and cellular mechanisms and therapeutic opportunities. J Scleroderma Relat Disord 2:137-152
2. van Laar JM, Varga J (2015) The immunopathology of systemic sclerosis. Semin Immunopathol 37:439-441

3. Matucci-Cerinic M, Krieg T, Guillevin L, Schwierin B, Rosenberg D, Cornelisse P, Denton CP (2016) Elucidating the burden of recurrent and chronic digital ulcers in systemic sclerosis: long-term results from the DUO Registry. Ann Rheum Dis 75:1770-1776

4. Allanore Y, Denton CP, Krieg T, Cornelisse P, Rosenberg D, Schwierin B, DUO Investigators et al (2016) Clinical characteristics and predictors of gangrene in patients with systemic sclerosis and digital ulcers in the Digital Ulcer Outcome Registry: a prospective, observational cohort. Ann Rheum Dis 75:1736-1740

5. Mihai C, Landewé R, van der Heijde D, Walker UA, Constantin PI, Gherghe AM, Ionescu R, Rednic S, Allanore Y, Avouac J, Czirják L, Hachulla E, Riemekasten G, Cozzi F, Airò P, Cutolo M, MuellerLadner U, Matucci-Cerinic M, EUSTAR co-authors (2016) Digital ulcers predict a worse disease course in patients with systemic sclerosis. Ann Rheum Dis 75:681-686

6. Meunier P, Dequidt L, Barnetche T, Lazaro E, Duffau P, Richez C, Couzi L, Truchetet ME, Seneschal J, the FHU ACRONIM (2018) Increased risk of mortality in systemic sclerosis-associated digital ulcers: a systematic review and meta-analysis. J Eur Acad Dermatol Venereol 33:405-409. https://doi.org/10.1111/jdv.15114

7. Kowal-Bielecka O, Fransen J, Avouac J, Becker M, Kulak A, Allanore Y, EUSTAR Coauthors et al (2017) Update of EULAR recommendations for the treatment of systemic sclerosis. Ann Rheum Dis 76:1327-1339

8. Walker UA, Tyndall A, Czirják L, Denton C, Farge-Bancel D, Kowal-Bielecka O et al (2017) Clinical risk assessment of organ manifestations in systemic sclerosis: a report from the EULAR Scleroderma Trials And Research group database. Ann Rheum Dis 66:754-763

9. Suliman YA, Bruni C, Johnson SR, Praino E, Alemam M, Borazan N, Cometi L, Myers B, Khanna D, Allanore Y, Baron M, Krieg T, Herrick A, Afonso A, Distler O, Kafaja S, Denton CP, MatucciCerinic M, Furst DE (2017) Defining skin ulcers in systemic sclerosis: systematic literature review and proposed World Scleroderma Foundation (WSF) definition. J Scleroderma Relat Disord 2:115120

10. Amanzi L, Braschi F, Fiori G, Galluccio F, Miniati I, Guiducci S, Conforti ML, Kaloudi O, Nacci F, Sacu O, Candelieri A, Pignone A, Rasero L, Conforti D, Matucci-Cerinic M (2010) Digital ulcers in scleroderma: staging, characteristics and sub-setting through observation of 1614 digital lesions. Rheumatology (Oxford) 49:13741382

11. van den Hoogen F, Khanna D, Fransen J, Johnson SR, Baron M, Tyndall A, Matucci-Cerinic M, Naden RP, Medsger TA Jr, Carreira PE, Riemekasten G, Clements PJ, Denton CP, Distler O, Allanore Y, Furst DE, Gabrielli A, Mayes MD, van Laar J, Seibold JR, Czirjak L, Steen VD, Inanc M, Kowal-Bielecka O, Müller-Ladner U, Valentini G, Veale DJ, Vonk MC, Walker UA, Chung L, Collier DH, Csuka ME, Fessler BJ, Guiducci S, Herrick A, Hsu VM, Jimenez S, Kahaleh B, Merkel PA, Sierakowski S, Silver RM, Simms RW, Varga J, Pope JE (2013) Classification criteria for systemic sclerosis: an ACR-EULAR Collaborative Initiative. Arthritis Rheum 65:2737-2747

12. Denton CP, Krieg T, Guillevin L, Schwierin B, Rosenberg D, Silkey M, Zultak M, Matucci-Cerinic M, DUO Registry investigators (2012) Demographic, clinical and antibody characteristics of patients with digital ulcers in systemic sclerosis: data from the DUO Registry. Ann Rheum Dis 71:718-721

13. Wirz EG, Jaeger VK, Allanore Y, Riemekasten G, Hachulla E, Distler $\mathrm{O}$, Airò $\mathrm{P}$, Carreira PE, Tikly M, Vettori S, Balbir Gurman A, Damjanov N, Müller-Ladner U, Distler J, Li M, Häusermann P, Walker UA, EUSTAR coauthors (2016) Incidence and predictors of cutaneous manifestations during the early course of systemic 
sclerosis: a 10-year longitudinal study from the EUSTAR database. Ann Rheum Dis 75:1285-1292

14. Ferri C, Valentini G, Cozzi F, Sebastiani M, Michelassi C, La Montagna G et al (2002) Systemic sclerosis: demographic, clinical, and serologic features and survival in 1,012 Italian patients. Medicine 81:139-153

15. Hachulla E, Clerson P, Launay D, Lambert M, Morell-Dubois S, Queyrel V et al (2007) Natural history of ischemic digital ulcers in systemic sclerosis: single-center retrospective longitudinal study. J Rheumatol 34:2423-2430

16. Tiev KP, Diot E, Clerson P, Dupuis-Simeon F, Hachulla E, Hatron PY et al (2009) Clinical features of scleroderma patients with or without prior or current ischemic digital ulcers: post-hoc analysis of a nationwide multicenter cohort (ItinerAIR-Sclerodermie). J Rheumatol 36:1470-1476

17. Khimdas S, Harding S, Bonner A, Zummer B, Baron M, Pope J (2011) Associations with digital ulcers in a large cohort of systemic sclerosis: results from the Canadian Scleroderma Research Group registry. Arthritis Care Res 63:142-149

18. Silva I, Teixeira A, Oliveira J, Almeida I, Almeida R, Águas A, Vasconcelos C (2015) Endothelial dysfunction and nailfold videocapillaroscopy pattern as predictors of digital ulcers in systemic sclerosis: a cohort study and review of the literature. Clin Rev Allergy Immunol 49:240-252

19. Xu D, Li MT, Hou Y, Wang Q, Hu CJ, Song N, Zhao JL, Zeng XF, Zhang FC (2013) Clinical characteristics of systemic sclerosis patients with digital ulcers in China. Clin Exp Rheumatol 31(2 Suppl 76):46-49

20. Jaeger VK, Valentini G, Hachulla E, Cozzi F, Distler O, Airó P, Czirják L, Allanore Y, Siegert E, Rosato E, Matucci-Cerinic M, Caimmi C, Henes J, Carreira PE, Smith V, del Galdo F, Denton CP, Ullman S, de Langhe E, Riccieri V, Alegre-Sancho JJ, Rednic S, Müller-Ladner U, Walker UA, EUSTAR coauthors (2018) Smoking in systemic sclerosis: a longitudinal European Scleroderma Trials and Research Group Study. Arthritis Rheum 70:1829-1834. https://doi.org/10.1002/art.40557

21. Moinzadeh P, Riemekasten G, Siegert E, Fierlbeck G, Henes J, Blank N, Melchers I, German Network for Systemic Scleroderma et al (2016) Vasoactive therapy in systemic sclerosis: real-life therapeutic practice in more than 3000 patients. J Rheumatol 43:66-74

22. Pope J, Harding S, Khimdas S, Bonner A, Baron M, Canadian Scleroderma Research Group (2012) Agreement with guidelines from a large database for management of systemic sclerosis: results from the Canadian Scleroderma. J Rheumatol 39:524-531

23. Ntelis K, Solomou EE, Sakkas L, Liossis SN, Daoussis D (2017) The role of platelets in autoimmunity, vasculopathy, and fibrosis: implications for systemic sclerosis. Semin Arthritis Rheum 47:409 441

Publisher's note Springer Nature remains neutral with regard to jurisdictional claims in published maps and institutional affiliations.

\section{Affiliations}

Jelena Blagojevic ${ }^{1}$ - G. Abignano ${ }^{2,3} \cdot$ J. Avouac $^{4} \cdot$ L. Cometi ${ }^{1}$ - M. Frerix ${ }^{5} \cdot$ S. Bellando-Randone ${ }^{1} \cdot$ S. Guiducci ${ }^{1}$.

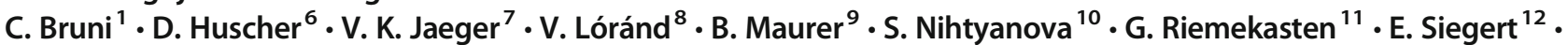

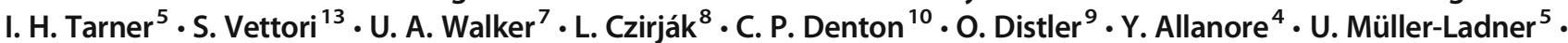
A. Moggi-Pignone ${ }^{14} \cdot$ M. Matucci-Cerinic ${ }^{1} \cdot$ F. Del Galdo $^{3}$

1 Department of Experimental and Clinical Medicine, Department of Geriatric Medicine, Division of Rheumatology and Scleroderma Unit AOUC, University of Florence, Florence, Italy

2 Rheumatology Institute of Lucania (IReL), Rheumatology Department of Lucania, San Carlo Hospital of Potenza and Madonna delle Grazie Hospital of Matera, Potenza, Italy

3 NIHR Leeds Biomedical Research Centre, Leeds Teaching Hospitals NHS Trust and Leeds Institute of Rheumatic and Musculoskeletal Medicine, University of Leeds, Leeds, UK

4 Department of Rheumatology, University of Paris Descartes, Paris, France

5 Department of Rheumatology and Clinical Immunology, Kerckhoff Klinik GmbH, Campus of the Justus-Liebig University Giessen, Bad Nauheim, Germany

6 Institute of Biometry and Clinical Epidemiology, Charité Universitätsmedizin Berlin, corporate member of Freie Universitaet Berlin, Humboldt-Universitaet zu Berlin, and Berlin Institute of Health, Berlin, Germany

7 Department of Rheumatology, University of Basel, Basel, Switzerland
8 Department of Rheumatology and Immunology, University of Pécs, Pecs, Hungary

9 Department of Rheumatology, University Hospital Zurich, Zurich, Switzerland

10 Department of Rheumatology, University College London, Royal Free Hospital, London, UK

11 Clinic of Rheumatology and Clinical Immunology, University of Lübeck, Lubeck, Germany

12 Department of Rheumatology and Clinical Immunology, Charité Universitaetsmedizin Berlin, corporate member of Freie Universitaet Berlin, Humboldt-Universitaet zu Berlin, and Berlin Institute of Health, Berlin, Germany

13 Rheumatology Section, Department of Precision Medicine, University of Campania “Luigi Vanvitelli", Naples, Italy

14 Department of Experimental and Clinical Medicine, Department of Emergency, Division of Medicine IV AOUC, University of Florence, Florence, Italy 